\title{
Metastasiertes Nierenzellkarzinom
}

\section{Wie gut sind die aktuellen Therapien?}

Das metastasierte Nierenzellkarzinom (mRCC) steht beispielhaft für den Therapiefortschritt in der Onkologie, der in den letzten Jahren durch zielgerichtete Therapien erreicht wurde. Auf dem ASCO 2011 in Chicago werden nеue interessante Daten zu dieser Indikation erwartet. Kurz vor dem Kongress hatten wir Gelegenheit, mit Prof. Manuela Schmidinger von der Medizinischen Universität Wien über die aktuelle Situation beim mRCC zu sprechen.

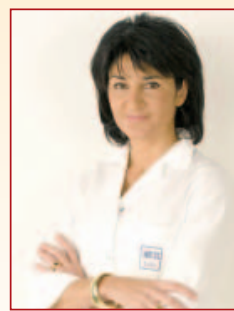

Interview mit Professo Manuela Schmidinger, Oberärztin der Klinik für Innere Medizin I der Abteilung für Onkologie an der Medizinischen Universität Wien

Frau Professor Schmidinger, wie hat sich die Therapie des $\mathrm{mRCC}$ in den letzten 5 Jahren verändert?

In der systemischen Therapie des mRCC wurde durch die Einführung zielgerichteter Substanzen ein Paradigmenwechsel eingeleitet, der die Situation von Patienten mit $\mathrm{mRCC}$ deutlich verbessert hat. Etablierte Therapien wie der Tyrosinkinase-Inhibitor (TKI) Sunitinib $\left(\right.$ Sutent $\left.{ }^{\circledR}\right)$ stellen heute einen anerkannten Standard dar, mit dem wir inzwischen auf 5 Jahre Erfahrung zurückblicken können. Auch wir Behandler sind durch diese positive Entwicklung in einer viel besseren Situation als früher: Mittlerweile stehen uns 6 zielgerichtete Medikamente mit teilweise unterschiedlichen Wirkmechanismen in der Firstund Second-Line-Therapie des mRCC zur Verfügung. Auf der anderen Seite haben wir dadurch im klinischen Alltag die «Qual der Wahl».

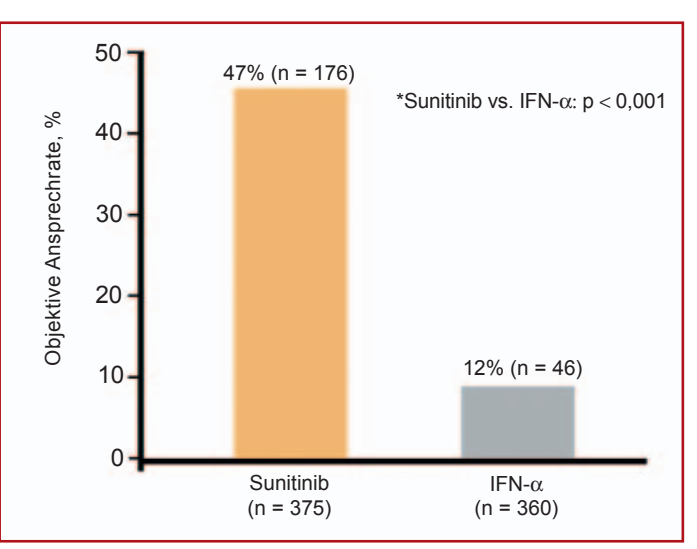

Worin zeigt sich die Verbesserung der Therapiesituation?

Mit den neuen Therapien sehen wir viel bessere Ansprechraten als unter der - inzwischen fast verdrängten - Therapie mit Zytokinen. Diese liegen bei einigen Substanzen bei etwa 30\%, Sunitinib erreichte in der Phase-III-Zulassungsstudie eine Ansprechrate von 47\% (Abb. 1) [1-6]. Das progressionsfreie Überleben liegt bei den FirstLine-Optionen Sunitinib, Bevacizumab + IFN und Pazopanib bei jeweils etwa 10-11 Monaten bei Patienten mit niedrigem und intermediärem Risiko. Sequenztherapien sind heute möglich. Das Gesamtüberleben hat sich von etwa 12 Monaten unter der Immuntherapie mit den zielgerichteten Therapien verdoppelt [2, 3, 7]. Mit Sunitinib konnte in der Phase-III-Studie ein Gesamtüberleben von 26,4 Monaten erreicht werden [4]. Insgesamt verfügen wir bei Sunitinib über eine sehr valide und umfassende Datenbasis. Die überzeugenden klinischen Studiendaten wurden durch Erfahrungen aus der klinischen Praxis und das deutsche «Tumorregister Fortgeschrittenes Nierenzellkarzinom» bestätigt [6].

Sie haben von der "Qual der Wahl» gesprochen. Welche Überlegungen bestimmen die Therapieentscheidung?

Der wichtigste Grundsatz in der palliativen Situation lautet: Wie erreichen wir ein verlängertes Gesamtüberleben bei guter Lebensqualität? Wir haben zunehmend Hinweise darauf, dass sich das Ansprechen auf die Therapie positiv auf das Gesamtüberleben auswirken könnte [8,9]. Dies deckt sich auch mit unseren eigenen Erkenntnissen. Diese Daten werden derzeit unter den mRCC-Experten diskutiert und könnten durchaus einen Einfluss auf die initiale Therapie haben. Denn in der Konsequenz würde dies bedeuten, dass die Ansprechrate ein wichtiges Kriterium für unsere Therapieentscheidung in der First Line darstellt.

Wie geht es beim mRCC weiter? Welche neuen Herausforderungen sehen Sie in diesem Bereich?

Das wichtigste Ziel der Therapie besteht darin, die Anwendung der bereits zugelassenen Medikamente im

Abb. 1. Objektive Ansprechrate unter Sunitinib in der Phase-III-Zulassungsstudie nach [4]. klinischen Alltag weiter zu optimieren und damit das bestmögliche Therapieergebnis zu erreichen. Wenn sich die Remissionsrate tatsächlich als ein Parameter für ein langes Überleben erweist, müsste man als initiale Therapie eine Substanz wählen, die hier eine besonders hohe Wirksamkeit gezeigt hat. Natürlich geht die Forschung beim $\mathrm{mRCC}$ insgesamt weiter. Zahlreiche neue Substanzen befinden sich in der Entwicklung, zu denen wir beim ASCO 2011 mit interessanten Ergebnissen rechnen.

Welche Aufgaben stellen sich derzeit in der klinischen Praxis?

Aus den zuvor genannten Gründen kommt der Auswahl der Erstlinientherapie eine besondere Bedeutung zu, da bei gutem Ansprechen ein langer Verlauf erhofft werden kann. Hinsichtlich der verfügbaren Daten unterscheiden sich die zugelassenen Substanzen in diesem Punkt, wobei die Datenlage für Sunitinib mit einem Ansprechen von 47\% wirklich sehr beeindruckend ist, so dass wir in dieser Situation meist Sunitinib einsetzen. Auf diese Weise konnten auch in unserer Klinik häufig Patienten über mehrere Jahre erfolgreich therapiert werden. Dabei ist ein adäquates Therapiemanagement von Anfang an sehr wichtig. Basis hierfür ist das Zusammenspiel von adäquater Dosierung, ausreichend langer Behandlungsdauer, effektiver Prävention und Therapie von Nebenwirkungen.

\section{Anne Krampe-Scheidler, Berlin}

\section{Referenzen}

Rini B et al.: J Clin Oncol 2009;27(suppl 15):18s, A-LBA5019.

2 Escudier B et al.: J Clin Oncol 2007;25(18s):3

3 Sternberg C et al.: J Clin Oncol 2010;28:1061-1098

4 Motzer RJ et al.: J Clin Oncol 2009;27:3584-3590.

5 Gore ME et al.: Lancet Oncol 2009;10:757-763.

6 Müller L et al:: DGU 2010, Vortragssitzung 19.

7 Motzer RJ et al.: J Clin Oncol 2002;20:289-296.

8 Golshayan AR et al.: J Clin Oncol 2009;27:15s(suppl abstr 5043).

9 Basappa N et al.: Cancer 2010. DOI: 10.1002/ cncr.25713.

\section{Impressum}

Metastasiertes Nierenzellkarzinom Wie gut sind die aktuellen Therapien? PharmaForum in OnKologie 34 | 6 | 11 (c) 2011 by S. Karger Verlag für Medizin und Naturwissenschaften $\mathrm{GmbH}$ Wilhelmstraße 20A 79098 Freiburg, Deutschland

Mit freundlicher Unterstïtzung durch Pfizer Pharma GmbH.

Verlag, Herausgeber, Redaktion und Verlagsgeschäftsführung übernehmen keine Verantwortung für den Inhalt dieser Rubrik. 


\section{Adjuvante Therapie des Kolonkarzinoms (UICC-Stadium III) \\ Mit Capecitabin/Oxaliplatin (XELOX) effektiv behandeln}

Für Patienten mit lokal fortgeschrittenem Kolonkarzinom im Stadium III (T3-4, N1-2) ist die Kombination Capecitabin/Oxaliplatin (XELOX) eine effektive und im klinischen Alltag gut handhabbare Therapieoption.

Bei Patienten mit Kolonkarzinom im Stadium III ist postoperativ eine adjuvante Fluoropyrimidinbasierte Kombinationschemotherapie mit Oxaliplatin Standard. Lediglich bei Kontraindikationen gegen Oxaliplatin sowie bei klinisch relevanten Komorbiditäten ist eine FluoropyrimidinMonotherapie - vorzugsweise mit einem oralen Fluoropyrimidin - indiziert [1].

\section{XELOX - effektive Kombinations- therapie}

Eine effektive Kombinationsbehandlung für Patienten im Stadium III ist das XELOX-Regime mit dem oralen Fluoropyrimidin Capecitabin plus Oxaliplatin. In der XELOXA-Studie [2], einer randomisierten Phase-III-Studie, war XELOX der 5-FU/FS-Bolus-Gabe beim primären Studienendpunkt, der krankheitsfreien Überlebenszeit (DFS), statistisch signifikant überlegen $(\mathrm{HR}=0,80 ; \mathrm{p}=0,0045)$. Nach 5 Jahren betrug das DFS bei XELOX $66,1 \%$ und bei 5-FU/FSBolus 59,8\%. Somit zeigte sich ein absoluter Vorteil für XELOX. Beim Gesamtüberleben (OS) zeigte sich nach 5 Jahren ein Trend zur Überlegenheit (HR 0,87; $p=0,1486$ ) für XELOX. Unbedenklich ist, dass im Kontrollarm ein 5-FUBolus-Regime eingesetzt wurde, da dieses genauso wirksam ist wie die infusionale 5-FU-Gabe und lediglich schlechter vertragen wird [3].

\section{XELOX gleich wirksam wie FOLFOX und FLOX}

Direkte Vergleichsstudien der verschiedenen Fluoropyrimidin-basierten Kombinationsregime gibt es nicht. Im indirekten Vergleich zeigen sich jedoch vergleichbare Wirksamkeitsergebnisse. Im Rahmen eines «Cross-trial-Vergleichs» [4] wurden 672 FOLFOX4-Patienten aus der MOSAIC-Studie mit 938 XELOX-Patienten aus der XELOXA-Studie verglichen. Es handelte sich jeweils um Patienten im Stadium III. Sowohl beim DFS als auch beim OS zeigten sich fast deckungsgleiche KaplanMeier-Kurven (Abb. 1).

Eine aktuelle Metaanalyse [5] mit über 9000 Patienten mit Kolonkarzinom im Stadium II/III weist die 3 Kombinationsregime - FOLFOX, FLOX und XELOX - ebenfalls als äquieffektiv aus. Außerdem untermauert der indirekte Vergleich der Studiendaten der großen randomisierten Phase-III-Studien X-ACT, XELOXA, MOSAIC (nur Stadium-III-Patienten), NSABPC07 und NO147 die vergleichbare Wirksamkeit der Regime XELOX und FOLFOX.

Die indirekten Studienvergleiche können in diesem Fall als legitim gelten, da die Ergebnisse sehr kongruent sind und auf langen Nachbeobachtungszeiten mit großen Patientenzahlen und gut vergleichbaren Patientenkollektiven beruhen.

\section{Nebenwirkungen sicher handhaben}

Unterschiede zwischen den Regimen zeigten sich lediglich bei den Nebenwirkungen [4]. Hier schnitt das XELOX-Regime vergleichsweise günstig ab: Im Vergleich zu den 5-FU/FS-basierten Kombinationen traten unter XELOX deutlich weniger Grad 3-4 Neutropenien auf. Grad 3-4 Diarrhöen waren zwar häufiger, doch hat die orale Gabe von Capecitabin hier den Vorteil, dass sich durch eine rechtzeitige Unterbrechung der Tabletteneinnahme die Diarrhö-Dauer kurz halten lässt (sog. Real-time-Kontrolle). Die Patienten müssen entsprechend aufgeklärt sein, so dass sie wissen, ob bzw. wann sie die Einnahme

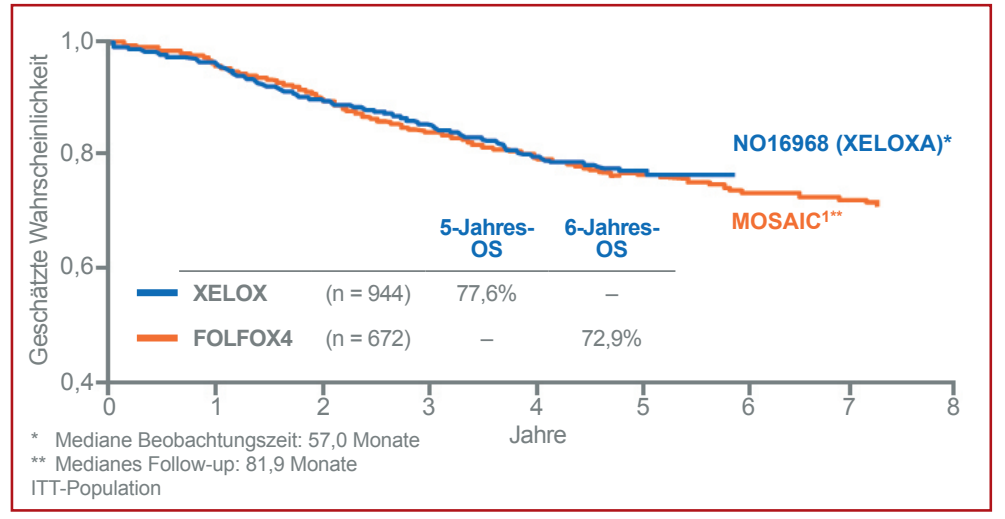

der Tabletten unterbrechen und sich beim Arzt melden müssen. Das für Capecitabin typische Hand-Fuß-Syndrom (HFS) trat unter XELOX bei nur 5,4\% (Grad 3-4) der Patienten auf.

\section{Individuelle Dosisanpassung wichtig}

Dosiskompromisse sollten in der adjuvanten Therapiesituation vermieden werden. Davon zu unterscheiden ist eine individuelle Dosisanpassung, die gerade bei der oralen Behandlung eine Rolle spielt und die Verträglichkeit erhöht. Dazu gehört, bei Auftreten einer klinisch relevanten Nebenwirkung die tägliche Capecitabin-Dosis zu deeskalieren oder vorübergehend abzusetzen, um mit einer niedrigeren Dosis erneut zu beginnen. Aktuelle Daten zum XELOX-Regime zeigen, dass es bei individuell angepasster Dosismodifikation zu keinem Wirksamkeitsverlust kommt $[2,6]$. Für das XELOX-Regime spricht außerdem, dass viele Patienten die orale Einnahme bevorzugen, da weniger Ambulanzbesuche und geringere Reisewege anfallen. Für die Patienten bedeutet dies mehr Flexibilität und Unabhängigkeit. Da XELOX ohne Port appliziert werden kann, entfallen außerdem Port-assoziierte Komplikationen [7].

Fazit: Ist eine Fluoropyrimidin-haltige Kombination indiziert, bietet XELOX eine effektive und flexible Therapie.

Birgit-Kristin Pohlmann, Nordkirchen

\section{Quelle}

Expertenroundtable: Adjuvante Therapie des Kolonkarzinoms, Frankfurt/Main, 13.12.2010

\section{Referenzen}

1 Schmiegel W et al.: Z Gastroenterol 2008;46:799-840. 2 Haller D et al.: JCO 2011;29(11):1465-71.

3 Carrato A et al.: JCO 2006;Proc ASCO Vol 24(18):3563a 4 Haller D et al.: ESMO 2009:LBA5 + oral presentation.

5 Cassidy J et al.: ASCO-GI 2011;498a.

6 Cassidy J et al.: ASCO-GI 2011;497.

7 Scheithauer W et al.: JCO ASCO 2007;4098 + oral presentation.
Abb. 1.

Cross-TrialVergleich zum Gesamtüberleben unter XELOX (XELOXAStudie) und FOLFOX4 (MOSAICStudie) [4].
Impressum

Expertenroundtable zur adjuvanten Therapie des Kolonkarzinoms (UICC-Stadium III)

Mit Capecitabin/Oxaliplatin (XELOX) aktive

Patienten effektiv behandeln

PharmaForum in ONKOLOGIE 34 | 6 | 11

(c) 2011 by S. Karger Verlag für Medizin und Naturwissenschaften $\mathrm{GmbH}$

Wilhelmstraße 20A

79098 Freiburg, Deutschland

Mit freundlicher Unterstützung durc Roche Pharma AG.

Verlag, Herausgeber, Redaktion und Verlagsgeschäftsführung übernehmen keine Verantwortung für den Inhalt dieser Rubrik. 